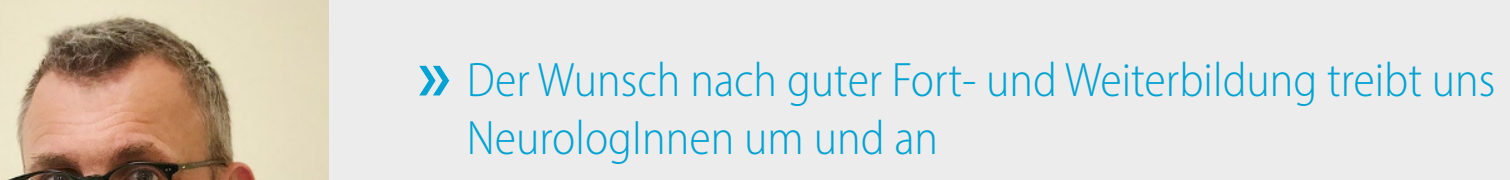

PD Dr. G. J. Jungehülsing

Klinik für Neurologie,

Jüdisches Krankenhaus Berlin

Heinz-Galinski-Straße 1, 13347 Berlin-Mitte, Deutschland

jan.jungehuelsing@dgn.org

\title{
Über Weiterbildung in Teilzeit oder von Zeiten der Teilweiterbildung
}

Vor wenigen Wochen hatte ich ein Mitarbeitergespräch mit einer Assistenzärztin meiner Klinik. Im Gespräch sagte sie gleich einleitend: „Danke Herr Jungehülsing, dass ich so unkompliziert auf $80 \%$ reduzieren konnte. Ich merke, wie gut mir das tut. Jetzt habe ich auch endlich wieder Zeit und Kraft, was Medizinisches zu lesen. Zudem wollte ich Sie fragen, ob es o.k. ist, wenn ich an freien Tagen vielleicht in einer Praxis hospitiere, um mich mehr in der Elektrophysiologie weiterzubilden“. Zuerst habe ich mich über das Kompliment gefreut, dann aber haben mich ihre Worte sehr nachdenklich gemacht. Sie werfen Fragen auf. Mich beschäftigt dabei vor allem das Thema Weiterbildung.

Warum wollen immer mehr MitarbeiterInnen ihre Stellenanteile reduzieren? Ist das wirklich ein Generationenthema? Was läuft falsch, dass wir es in unseren Kliniken seit Jahren nicht ausreichend schaffen, in vielen Bereichen die Weiterbildung sicherzustellen? Was läuft falsch im System? Warum ist die Ausbildung von AssistenzärztInnen in der Niederlassung so wenig ein Thema? Warum sind bei Fragen der Fort- und Weiterbildung Niedergelassene und Klinik-NeurologInnen nicht mehr miteinander verzahnt?

Wie wichtig uns ÄrztInnen aller Altersgruppen und aus allen Bereichen das Thema Fort- und Weiterbildung ist, zeigen auch die Ergebnisse aus der crossmedialen ReImagine Medicine Initiative der Deutschen Gesellschaft für Neurologie (DGN). In Freitext-Antworten konnten über 1200 NeurologInnen benennen, was ihnen in Zukunft für die Weiterentwicklung des Arztund Neurologenberufs wichtig erscheint. Die Antworten wurden über die Methode Insights zu Erkenntnissen verdichtet.

Es zeigt sich, dass es weniger ökonomische Sachzwänge zu sein scheinen, die uns Neurologen umtreiben, als vielmehr die Sorge um bzw. der Wunsch nach guter Fort- und Weiterbildung. Es wurde deutlich, wie wichtig uns ÄrztInnen die Weiterentwicklung der Weiterbildung ist, z. B. indem neue Formate etabliert werden. Die Teilnehmer der Umfrage diskutieren eine stärkere Förderung externer Weiterbildung, regelmäßige Kurzfortbildungen in Kliniken und Praxen, eine bessere Verfügbarkeit von

DGNeurologie $2020 \cdot 3(1): 1-2$

https://doi.org/10.1007/s42451-019-00145-w

(c) Springer Medizin Verlag GmbH, ein Teil von Springer Nature 2020
Fachliteratur und die Frage, wie sich die Potenziale der Digitalisierung für Fort- und Weiterbildung besser nutzen ließen. Drei Aspekte scheinen besonders interessant.

Erstens: Immer wieder fordern KollegInnen eine Weiterentwicklung der (Facharzt-)Ausbildung, die die Veränderungen der Neurologie in den letzten Jahrzehnten stärker berücksichtigt. Viele AssistentInnen in Ausbildung wünschen sich Rotationen in die Innere Medizin, die Neuroradiologie und die Neurochirurgie und eine echte fundierte intensivmedizinische Grundausbildung (und kein „Stroke-Unit-Feigenblatt“ im Facharztzeugnis). Gleichzeitig werden eine stärkere Strukturierung des Ausbildungscurriculums mit vielfältigeren fachübergreifenden und strukturierten Rotationen, ein z. B. auch von der DGN begleitetes Online-Curriculum und regelmäßige „Bedside Teachings“ durch erfahrene KollegInnen gewünscht.

Das bringt mich zum zweiten Punkt, dem Mentoring in der Ausbildung. Wir alle wissen, wie entscheidend eine gute Betreuung in der Facharztausbildung ist. Fach-, Ober- und ChefärztInnen sollten im Arbeitsalltag Zeit haben, die AssistenzärztInnen zu unterrichten und anzuleiten. Hier liegt das Problem, denn mit höherer zeitlicher Verdichtung und oft stärkeren ökonomischen Zwängen fehlt zuerst die Zeit für Ausbildung - nicht zuletzt, weil Ausbildung im INEK-gesteuerten EBM- und DRG-Erlössystem nicht berücksichtigt ist. Also müssen wir dafür kämpfen, dass auch Ausbildung zeitlich und finanziell erstattet wird. Auch neue Mentorenprogramme für junge AssistenzärztInnen sind überlegenswert: Warum können nicht zum Beispiel ÄrztInnen im Ruhestand stärker in die Ausbildung einbezogen werden und so einen Wissenstransfer zu weniger erfahrenen KollegInnen fördern?

Eine dritte sehr spannende Erkenntnis aus ReImagine Medicine ist, dass viele sich einen intensiveren Austausch zwischen verschiedenen Einrichtungen wünschen. Zum Beispiel sollten Kliniken und Praxen ihre Zusammenarbeit hinsichtlich Weiterbildung verbessern. Außerdem könnten die Stärken unterschiedlicher Kliniken und Praxen mit besonderen Schwerpunkten über regional aufeinander abgestimmte Weiterbildungsprogramme stärker genutzt werden.

Auch wenn sich Medizin und Neurologie in den letzten Jahrzehnten stark verändert haben, und damit insbesondere wir KrankenhausneurologInnen nach einer Neufassung der Weiterbildungsordnung hin zu mehr akutmedizinischen Inhalten (und weniger Psychiatrie) rufen: Wir dürfen nicht vergessen, dass ein 
großer Teil von uns NeurologInnen ambulant tätig ist bzw. später sein wird. Und dieser Teil mit einer zunehmenden Verlagerung bestimmter Neuro- und Psychiatrie-Inhalte in den ambulanten Bereich wird in Zukunft sicher nicht kleiner.

Zurück zum Gespräch mit meiner Mitarbeiterin und der Frage, ob wir es vielleicht mit einer vielleicht besonders anspruchsvollen Generation zu tun haben. Ich denke, das haben wir nicht, und ich habe persönlich ein Lamento über die Generationen Y oder Z schon immer für wenig hilfreich gehalten. Jede Generation hat das Zusammen- und das Arbeitsleben geändert [1]. Früher war nie alles besser, eher schlimmer. Auch über meine Generation X bzw. Golf [2] oder die vorherigen Babyboomer wurde sicher mal geschimpft. Arbeit hat sich massiv verändert und verdichtet. Die Zahl der PatientInnen ist gestiegen, der Aufwand der Dokumentation ist größer und die Neurologie als Ganzes sehr viel umfassender geworden. Die Digitalisierung der Menschheit - auch wenn wir in der Medizin noch ziemlich weit davon entfernt sind - hat nicht alles einfacher gemacht, im Gegenteil. Heute befinden wir uns oft viel mehr in CC-E-Mail-Beschäftigungsschleifen, verbringen Zeit mit digitaler Non-Kommunikation, wo ein kurzer Anruf die Lösung gewesen wäre, und sind so datengeschützt, dass es wehtut. Und nur am Rande sei erzählt, dass wir vor kurzem ein großes IT-Update in unserer Klinik hatten. Gut vorbereitet haben wir dann an diesem Wochenende fröhlich Untersuchungen auf Papier angefordert, in echten StrokeKurven mit Kugelschreiber dokumentiert und häufiger primär miteinander gesprochen und telefoniert. Schlimm war das nicht.

Was können wir also tun? Am besten, wir fangen bei uns selbst an. Geben wir uns nicht zufrieden, die Intensiv-Weiterbildung einfach zu bestätigen, wenn ein/e AssistentIn „nur“ auf der Stroke Unit war oder für ein paar Wochen die Konsiliarärztin/den Konsiliararzt auf der Intensivstation begleitet hat. Setzen wir die Funktion Elektrophysiologie in Dienstplan und Realität so durch, dass diese Ärztin oder dieser Arzt nicht ständig als Springer eingesetzt werden kann und darunter die ohnehin knapp bemessene Ausbildung leidet. Werben und kämpfen wir dafür, dass Oberund FachärztInnen nicht auch noch in Administration ersticken, sondern besser Zeit mit der Weiterbildung der AssistentInnen verbringen. Lassen wir z. B. Zertifizierungsprozesse von der Verwaltung so unterstützen, dass möglichst wenig bei den OberärztInnen hängen bleibt. Verstärken und institutionalisieren wir in vielen Bereichen - von Behandlung bis Weiterbildung - Kooperationen zwischen Kliniken und niedergelassenen KollegInnen. Kämpfen wir als Fachgesellschaft dafür, dass Ausbildung auch finanziell erstattet, also im DRG-System oder besser: von ihm erlöst wird.

Wenn wir über Weiterbildung sprechen, dann geht es um Wertschätzung (und Gewinnung!) von MitarbeiterInnen. Vor allem aber geht es um unsere PatientInnen und um nicht weniger als die Zukunft der Medizin. Schließlich wollen wir Generationen $\mathrm{X}$ und Babyboomer auch einmal gut von den Ypsi- und Z-lern und allen, die danach kommen, behandelt werden.

Ich wünsche Ihnen alles Gute für das neue Jahr 2020, Gelassenheit und stetige Fortbildung!

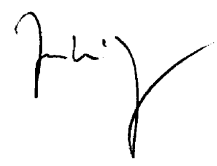

Ihr Gerhard Jan Jungehülsing
Interessenkonflikt. G.J. Jungehülsing gibt an, dass kein Interessenkonflikt besteht.

\section{Literatur}

1. Kerstin Bund: GENERATION Y - Wir sind jung... und brauchen das Glück: Wie die Generation Y die Berufswelt verändert und warum alle von diesem Wandel profitieren. ZEIT online 27. Februar 2014.

2. Illies F (2000) Generation Golf, Eine Inspektion. Fischer-Taschenbuch Verlag. 